\title{
Diagnóstico y tratamiento de hipomineralización molar primaria en gemelos: reporte de un caso
}

\author{
Carolina Vansan, ${ }^{1}$ \\ Luis Furlan, ${ }^{2}$ \\ José Carlos Pettorossi Imparato, ${ }^{3}$
}

\section{Resumen}

Los defectos del esmalte dental en la dentición primaria en niños con antecedentes de prematuridad y bajo peso son frecuentes. Una de las causas de la prematuridad es el embarazo gemelar, que hace que la mayoría de los fetos sean prematuros debido al crecimiento intrauterino restringido. El objetivo de este trabajo es informar de la importancia de un diagnóstico correcto y precoz de la hipomineralización de los molares deciduos (HMD) en gemelos prematuros, así como de las posibilidades de tratamiento. Materiales y métodos: Dos niñas gemelas idénticas de 5 años que presentaban el mismo patrón de HMD en los dientes $55,65,75,85$ ya con gran destrucción e historia de sensibilidad. Ya habían recibido atención odontológica, pero sólo una de las hermanas recibió tratamiento restaurador, la segunda no permitió el tratamiento, se utilizaron técnicas de manejo de la conducta, pero no fue posible realizar procedimientos operatorios. En el examen clínico y radiográfico se constató la posibilidad de realizar la técnica de Hall en los dientes 55,65 de la gemela 1 y 85 de la gemela 2 . Resultados: Los dientes 75 y 85 de 1 a gemela 1, fueron diagnosticados clínica y radiográficamente con necesidad de restauración en resina compuesta, ya que estaban con restauraciones provisionales y asintomáticos. Los dientes 55 y 65 de la gemela 2 recibieron restauraciones ionoméricas y el diente 75 recibió una pulpectomía y una corona de acero. Conclusión: La técnica de Hall es muy bienvenida cuando se trata de rehabilitaciones en hipoplasia, especialmente cuando se trata del manejo de la edad joven, todavía hay diagnósticos erróneos cuando se examina una HMD (hipomineralización molar primaria).

Palabras clave: Hipoplasia del esmalte dental, Prematuros, Odontología Pediátrica, Embarazo de gemelos, Diente primario.

\footnotetext{
${ }^{1}$ Dentista pediátrico, ortodoncista, estudiante de maestría em odontología pediátrica.

${ }^{2}$ Dentista pediátrico, Máster em Odontología pediátrica en SLM.

${ }^{3}$ Profesor y Profesor de Odontología Pediátrica en La Universidad de São Paulo, profesor y coordinador del programa de posgrado de São Leopoldo Mandic.
} 


\section{Diagnóstico e tratamento de hipomineralização molar decíduo em gêmeos: Relato de caso}

\section{Resumo}

Defeitos de esmalte dentário na dentição decídua são comuns em crianças com histórico de prematuridade e baixo peso. Uma das causas da prematuridade é a gestação gemelar, esta faz com que a maioria dos fetos sejam prematuros devido ao crescimento intrauterino restrito. $\mathrm{O}$ objetivo deste trabalho é relatar a importância de um diagnóstico correto e precoce de Hipomineraliação Molar Decíduo (HMD) em gêmeos prematuros, e as possibilidades de tratamento. Materiais e Métodos: duas crianças 5 anos sexo feminino gêmeas univitelinas que possuíam o mesmo padrão de HMD nos dentes 55,65,75,85 já com grande destruição e histórico de sensibilidade. As mesmas já receberam atendimento odontológico, porém apenas uma das irmãs recebeu tratamento restaurador, a segunda irmã não permitiu tratamento, técnicas de gestão comportamental foram utilizadas, mas não foi possível a realização dos procedimentos operatórios. Em exame clínico e radiográfico constatou-se a possibilidade de execução da técnica Hall nos dentes 55,65 da gêmea 1 e 85 da gêmea 2. Resultados: Os dentes 75 e 85 da gêmea 1, foram diagnosticados clinicamente e radiograficamente com necessidade restauradoraem resina composta,pois estavam com restaurações provisórias e assintomáticos. Os dentes 55 e 65 da gêmea 2 receberam restaurações ionoméricas e o dente 75 pulpectomia e coroa de aço. Conclusão: A Hall Technique é muito bemvinda quando se trata de reabilitações em hipoplasias, principalmente no que tange ao manejo de pouca idade, ainda existem erros de diagnóstico na hora de examinar uma HMD (Hipomineralização Molar Decíduo).

Palavra-chave: Hipoplasia do esmalte dentário; Prematuros, Odontopediatria, Gravidez gemelar, Dente decíduo.

Case report

\section{Diagnosis and treatment of deciduous molar hypomineralization in twins: Case report}

\begin{abstract}
Dental enamel defects in the primary dentition are common in children with a history of prematurity and low birth weight. One of the causes of prematurity is twin pregnancy, which causes most cases to be premature due to restricted intrauterine growth. The objective of this report is to relate the importance of a correct and early
\end{abstract}

diagnosis and as treatment alternatives for of Deciduous Molar Hypomineraliation $(\mathrm{DMH})$ in premature twins. Materials and methods: 5 years old, female, identical twins who had the same HMD pattern in their teeth 55,65,75,85 presented with a long history of destruction and sensitivity. They had previously received dental care. Only one of the sisters received restorative treatment. The other sister did not have 
treatment performed although, behavioral management techniques were used. In clinical and radiographic examination, the possibility of performing the Hall technique on teeth 55, 65 of female 1 and 85 of female 2 was found. Results: Teeth 75 and 85 of female 1 were diagnosed clinically and radiographically with restoration restored in composite resin., as they had provisional and asymptomatic restorations. Teeth 55 and 65 of twin 2 receive ionomeric

\section{Introducción}

El nacimiento prematuro de bebés ha sido mencionado comocausa de malformaciones del esmalte dentario, en lo que concierne a alteraciones en el color dental, que es un defecto de carácter cualitativo y en alteraciones de carácter cuantitativo, como alteraciones hipoplásicas, lo que puede causar mucha molestia a los niños. ${ }^{1}$ Se sabe que algunos disturbios del desarrollo del esmalte dentario en la dentición decidua están relacionados al nacimiento prematuro y sus consecuencias. Esto no ocurre solamente por razón del nacimiento precoz, sino a través de varias complicaciones asociadas a ella. ${ }^{2}$

La etiologia de HMD (Hipomineralización Molar Deciduo) no es muy difundida en el medio científico. Sin embargo, se sabe que factores ambientales $y$ genéticos pueden interferir directamente en la calidad de la matriz orgánica del esmalte. Además, algunos factores como: el embarazo gemelar, el tabaquismo materno, la fertilización in vitro; factores socioeconómicos; presencia de vitamina D al nacimiento; entre otros, pueden contribuir para originar el defecto cualitativo mencionado en el esmalte dentario de los molares primarios. ${ }^{3}$ restorations and tooth 75 pulpectomy and steel crown. Conclusion: Hall Technique is very welcome when it comes to rehabilitation in hypoplasia, especially with regard to the middle age, there are still diagnostic errors when examining an HMD (Deciduous Molar Hypomineralization).

Key words: Dental enamel hypoplasia, Premature, Pediatric dentistry pregnancy, Twin, Tooth, Deciduous.

Un diagnóstico correcto es esencial para un tratamiento efectivo. Luego, conocer los aspectos de la lesión hipoplásica y el defecto de desarrollo del esmate es imprescindible para la planificaión del tratamiento. El opacamiento demarcado en los dientes deciduos le causa al niño mucha incomodidad y debe ser tratado llevando en consideración factores como la larga duración restauradora y técnicas de manejo. En este contexto, la técnica de Hall, a través de las coronas de acero, han demostrado ser una óptima opción, pues no exige ningún tipo de preparación cavitaria, lo que reduce el tiempo total del procedimento. ${ }^{4}$

De esta manera, conservar la dentición decidua por medio de un correcto diagnóstico y disponer de técnicas minimamente invasivas en la odontopediatría es una excelente opción de tratamiento, cuando se piensa en la filosofía de mínima intervención. Siendo así, el tratamiento de las lesiones de caries en dientes deciduos depende de la profundidad de las lesiones y de la extensión de la superficie involucrada. No obstante, no existe evidencia científica que destaque el mejor tratamiento a ser realizado. ${ }^{5}$ Entre las opciones, se menciona el tratamiento hecho con coronas de acero 
pre-contorneadas, lo cual es más duradero, tanto en lesiones de caries e hipoplasias, como en la rehabilitación al tratamiento pulpar, al compararlo con otros materiales de relleno. ${ }^{6}$

Los tratamientos de las lesiones de caries dentinaria en dientes deciduos dependen del diagnóstico referente a la profundidad y extesión de estas lesiones. Sin embargo, aunque haya pocos estudios que presentan evidencias de tratamiento eficaz a respeto de técnicas restauradoras, la técnica de Hall destaca como una alternativa en el tratamiento infantil, pues, dentro de la filosofía de mínima intervención y en dientes sin señales clínicas y radiográficas de patologia pulpar, la técnica de Hall, se muestra bastante aceptada por los niños durante el manejo odontopediátrico. ${ }^{7}$

\section{Caso Clínico}

Ha sido estudiado el caso de dos niñas de 5 años, gemelas, univetelinas idénticas que poseían el mismo patrón de HMD en los dientes $55,65,75$ y 85 , que ya se encontraban con alto grado de destrucción. En la gemela 1, se ha constatado lesión de caries en los dientes 85, 75 y 65 (Figura 1). Por otra parte, en la gemela 2, la lesión se concentraba en los dientes 55 y 65 (Figura 2). Además, se observó en ambas gemelas un histórico de sensibilidad en los dientes 55 y 65, seguido de incomodidad provocada durante el cepillado de dientes. Se observó, también, que la destrucción coronal en los segundos molares superiores de las niñas era consecuencia de las fracturas con la exposición dentinaria y acumulación de biofilm que ocasionan lesiones cariosas.
Una de las gemelas, recibió tratamiento restaurador con otro profesional, pero no hubo éxito. La otra niña, no aceptó el condicionamiento, o sea, no sufrió ninguna intervención de esta naturaleza. En efecto, dicen sus padres que la principal queja de las hermanas es el dolor que sienten al masticar y cepillarse los dientes. Además, en la anamnesis de las condiciones generales e historial médico, el padre relató que la madre de las niñas presentó un embarazo complicado, con mucha medicación y reposo prolongado. Dijo, también, que nacieron prematuras por cesárea, así que necesitaron de hospitalización por algunas semanas para mejores cuidados, pero sin riesgo de muerte. Actualmente, las niñas no presentan ninguna secuela por causa del nacimiento prematuro. Por el contrario, poseen buena estatura y peso saludable, dentro de lo normal para sus edades. A continuación, el padre relató que la gemela 2 aún no había recibido ningún tratamiento odontológico, esta, sería su primera vez. Así, en la cita odontológica, por medio del exámen clínico y radiográfico se constató que había posibilidad de ejecutar la técnica Hall en los dientes 55 y 65 de la gemela 1 , y en el diente 85 de la gemela 2. Para comprender, la técnica (de Hall) se apoya en un abordaje biológico de la caries dental colocándose coronas metálicas precontorneadas sin preparación dentaria, sin remoción del tejido cariado y sin anestesia, con objetivo mínimamente invasivo.

En tal sentido, la gemela 1 recibió restauraciones en los dientes 75 y 85 en resina compuesta, pues como supradicho, ya había recibido restauraciones invasivas (Figura 3). La gemela 2 recibió restauraciones ionómero en los dientes 55 y 56 a través de la filosofía de ART 
(Tratamiento Restaurador Atraumático). También, en el diente 75, pulpectomía y corona de acero debido la gran destrucción coronal, procedimiento que proporciona mejor preservación del tratamiento endodóntico (Figura 4). Los criterios usados para la elección del tratamiento de las niñas están basados en evidencias clínicas y científicas de la mínima intervención del tratamiento de HMD.

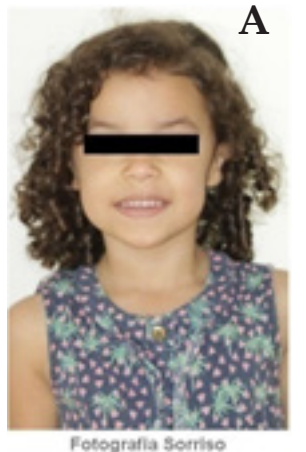

Figura 1. Gemela 1, una imagen clínica y radiológica sacada al iniciar el tratamiento. Se observa: en la imagen $A$, la fotografía
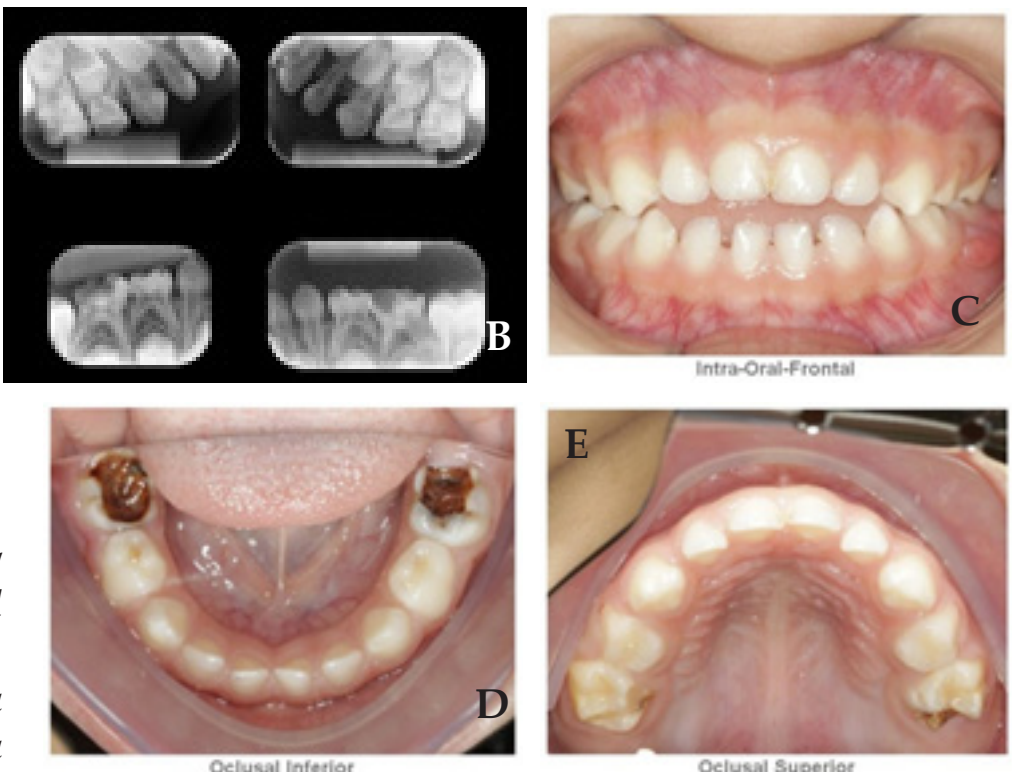

de la sonrisa; la imagen $B$, radiografías periapicales; en la figura $C$, imagen vestibular de los dientes superiores e inferiores; en las imágenes D y E muestran la situación intrabucales de las superficie oclusales de las arcadas inferiores y superiores. Además, se ve los dientes 75, 85, 55 y 65 con hipomineralización.
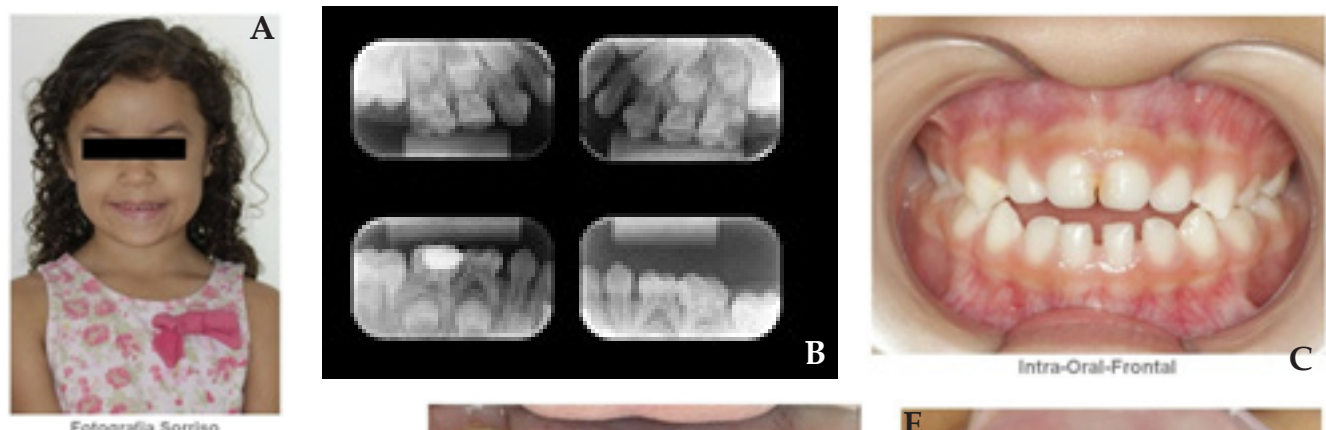

Fotografia Sorriso

Figura 2. Gemela 2: Imagen clínica y radiológica sacadas al iniciar el tratamiento. Se observa: en la imagen A, la fotografía de la sonrisa; en la $B$, radiografías periapicales;
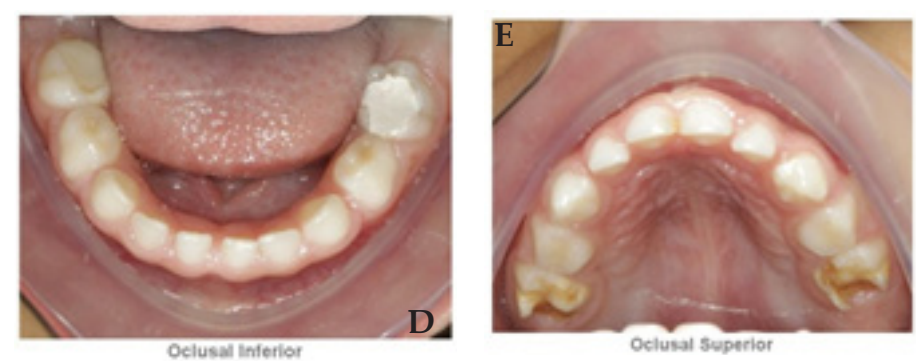

en la figura $C$, imagen vestibular de los dientes superiores e inferiores; las imágenes D y E muestran imágenes intrabucales de las superficie oclusales de las arcadas inferiores y superiores. Asemás, Se ve los dientes 55 y 65 con hipoplasia 

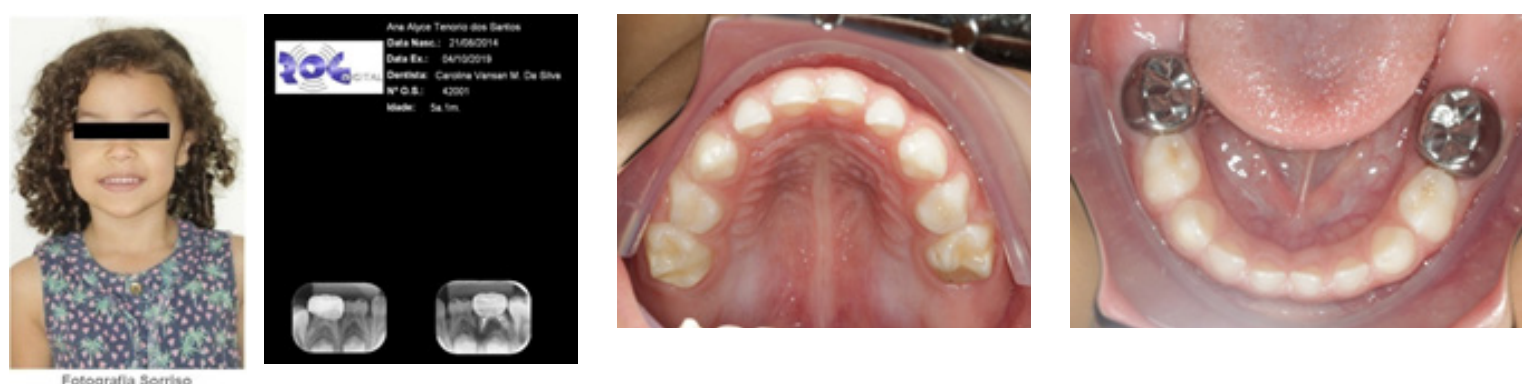

Figura 3. Gemela 1: Imagen clínica y radiológica sacadas al finalizar el tratamiento. Se observa: en la imagen 1, fotografía de la sonrisa; en la 2, radiografías periapicales en las cuales se ve una corona de acero en los dientes 75 y 78; en las imágenes 3 y 4 se muestra imágenes intrabucales de las caras oclusales de las arcadas inferiores y superiores. Se nota los tratamientos realizados: coronas de acero en los dientes 85 y 87, restauración con cemento de ionómero de vidrio en los dientes 55 y 65, además en los dientes 75, 85, 55 y 65 con hipomineralización.
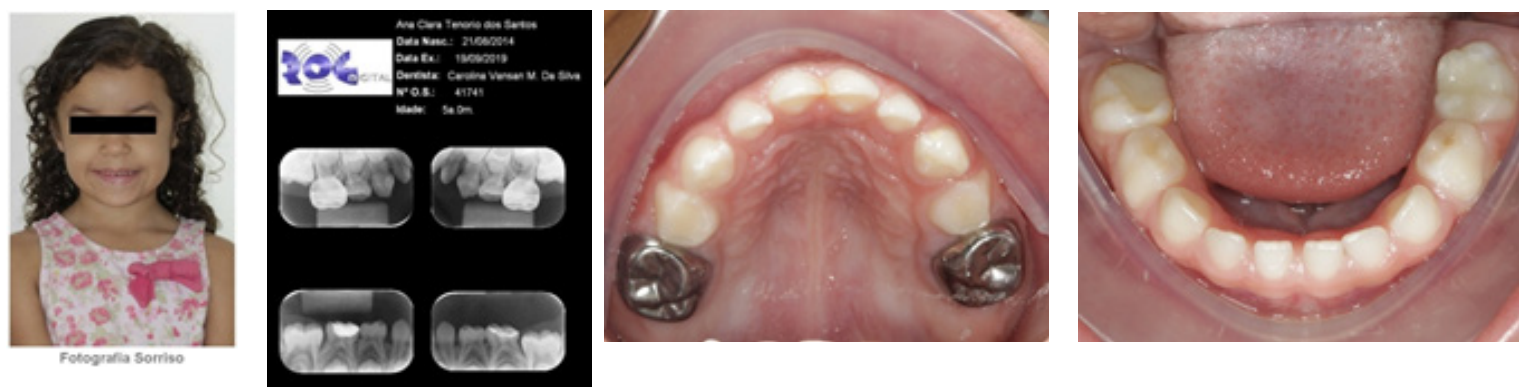

Figura 4. Gemela 2: Imágen clínica y radiológica sacadas al final del tratamiento. Se nota: en la imagen 1, fotografía de la sonrisa; en la 2 (dos), radiografías periapicales; en las imágenes 3 y 4 se ve imágenes intra bucales de las caras oclusales de las arcadas inferiores y superiores. Se observa que los dientes 55 y 56 tratados con coronas de acero y los dientes 75 y 85 restaurados con resina compuesta.

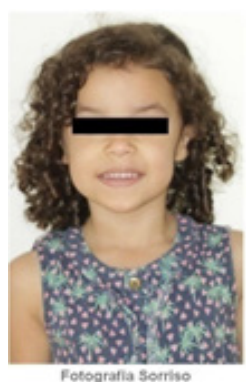

Gemela 1
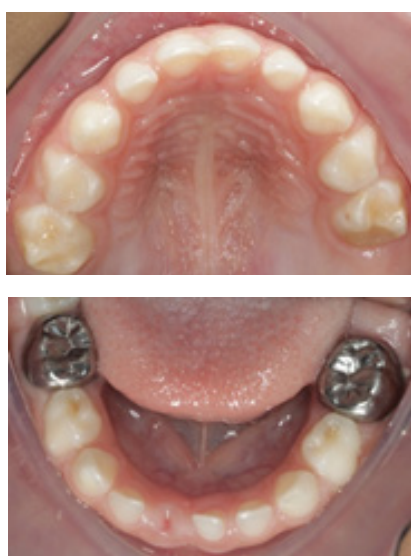

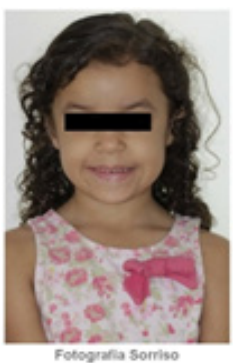

Gemela 2
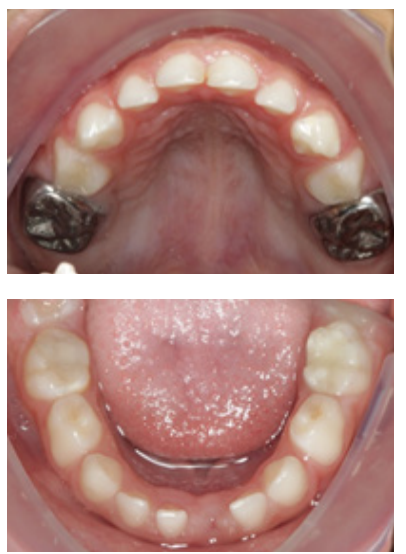

Figura 5. Monitoreo a los 6 meses 

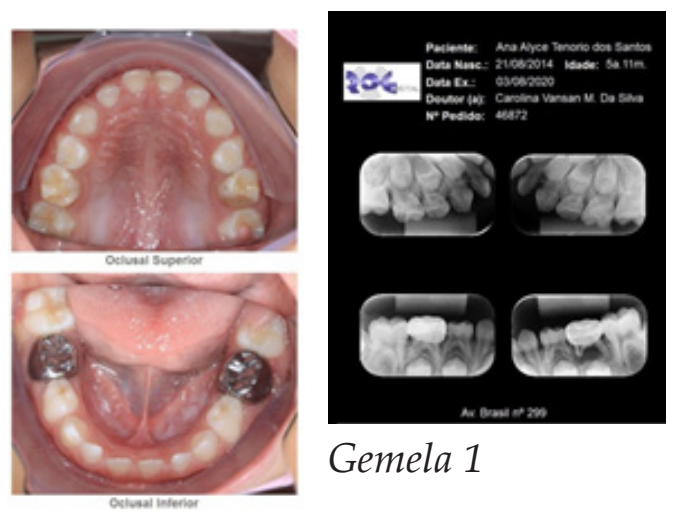

Gemela 1

Figura 6. Monitoreo a los 12 meses

\section{Discusión}

La prematurez es una de las causa de los defectos de esmaltes presentados en la dentición decidua. Los estudios realizados en bebés prematuros demuestran que la ocurriencia de estos defectos en neonatos con bajo peso es alta, ${ }^{2}$ además de presentar otras alteraciones como el retraso en la erupción dentaria.

Muchas complicaciones asociadas al nacimiento prematuro hacen que la falta de madurez de los órganos internos encargados de metabolizar el calcio como el hígado, riñones y glándulas de la paratiroides presenten alteraciones en su función. Debido a esto la matriz orgánica del esmalte dental se afecta, ya que necesita de ese mineral para formarse homogenemente. ${ }^{8}$

Diferentes estudios han demostrado que el uso de las coronas de acero en molares primarios es efectivo cuando se refiere al largo período de duración de la restauración, a la simplicidad de uso de la técnica; al tratamiento sin dolor; y a la facilidad de manejar tanto los materiales como a los niños. La combinación de las coronas de acero con la técnica de Hall,
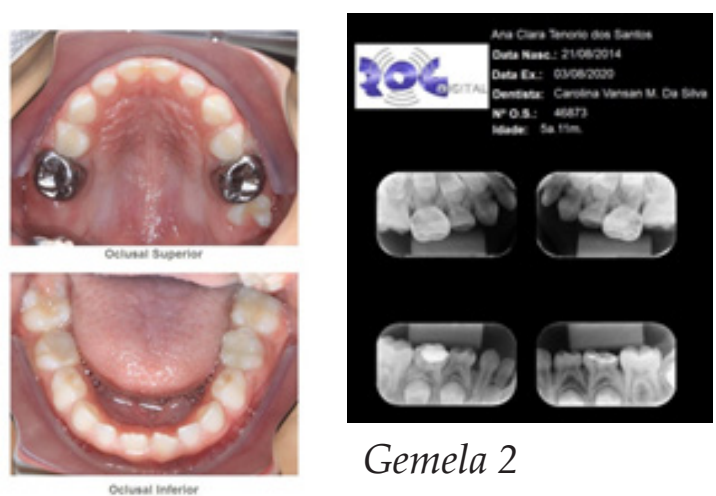

Gemela 2 como alternativa es superior si se compara con los demás métodos convencionales. ${ }^{7}$ usados en el tratamiento de las hipoplasias y/o hipomineralización de esmalte en la dentición decidua demostrando, una vez más, ser una opción eficaz cuando es asociada a la técnica de Hall, ya que no es necesario remover el tejido cariado.

Estudios retrospectivos a largo plazo han demostrado que las coronas metálicas utilizadas en la técnica de Hall poseen alta tasa de supervivencia en comparación a otros materiales. ${ }^{9}$ Otros autores, usaron materiales como: amálgama, cemento de ionómero de vidrio, cemento de ionómero de vidrio modificado por resina y resina compuesta, versus las coronas metálicas en restauraciones clase II. Concluyeron, que las coronas metálicas poseían mayor tasa de éxito a largo plazo debido a las limitaciones de los demás materiales. ${ }^{10,11}$ En el caso presentado, las coronas metálicas utilizadas en los molares deciduos de ambas las gemelas están de acuerdo con la literatura que fundamentó la búsqueda.

Los defectos del esmalte dentario pueden ser descritos como alteraciones en su estructura, los cuales pueden ser cualitativas o cuantitativas. De acuerdo 
con la OMS (Organización Mundial de Salud), esos defectos dentales deben seguir el índice DDE para que puedan ser clasificados y reconocidos. ${ }^{12}$ Sin embargo, la etiología de estos defectos puede estar asociada a factores hereditarios, que comprometen la maduración del esmalte y saberlo por medio de una buena anamnesis ayuda en la planificación del tratamiento, con el objetivo de minimizar las consecuencias como el malestar y la sensibilidad en los dientes afectados. ${ }^{13}$ Es importante destacar el uso de coronas de acero como mejor alternativa y de la técnica de Hall como una excelente opción para el manejo de las lesiones de HMD.

\section{Conclusión}

La atención odontopediátrica y sus desafíos induce al profesional del área a investigar enfoques terapéuticos que ofrezcan facilidad de manipulación, eficacia y larga duración restauradora. Así, en el caso clínico, objeto de este artículo, se optó por el enfoque de mínima intervención para tratar el defecto del esmalte de las niñas gemelas, lo que facilitó el trabajo con el manejo, además, proporcionó más tranquilidad a la família y se demostró muy eficaz a largo plazo.

\section{Referencias bibliográficas}

1. Seow WK. Enamel hypoplasia in the primary dentition: a review. J Dent Child1991 Oct; 58(6): 441-52.

2. Caixeta FF, CorrêaMSNP.Evaluation of the dental eruption pattern and of enamel defects in the premature child. RevAssocMedBras 2005; 51(4): 195-9.

3. Silva M J,Kilpatrick NM, Craig JM, Manton DJ, Leong P, Burgner D, Scurrah KJ. Etiology of hipomineralizad deciduous second molars: a study prospective twin. J Dent Res. 2019 Jan; 98(1): 77-83.

4. Gross DJ, Samways DM, ET AL. Hall technique in pediatric patients: case study and clinical-radiographic follow-up. Ver Bras Odontol. 2018; v. 75: 1-4.

5. Tedesco TK, Gimenez T, Floriano I, Montagner AF, Camargo LB, Calvo AFB, Morimoto S, Raggio DP. Scientific evidence for the management of dentin caries lesions in pediatric dentistry: Asystematic review and network meta-analysis. PloS One. 2018 Nov; 13(11).

6. Innes NP, Ricketts D, Chong LY, Keightley AJ, Lamont T, Santamaria RM. Preformed crowns for decayed primary molar teeth. Cochrane Database Syst Rev.2015 Dec; 31(12).

7. Santamaria R, Innes N, Machiulskiene V, Evans DJP, Alkiezy M, Splieth CH.Acceptability of different caries management methods for primary molars in a RCT. Jour Paed Dent. 2015; 25: 9-17.

8. Eid RMR. Questões do dia-a.dia. Prematuridade e defeitos do esmalte.Revista APCD 2001;5(3):32.

9. Grahnen H, Sjölin S, Stenström A. Mineralization defects of primary teethin children born pre-term. Scand J Dent Res 1974;82(5):396-400.

10. Alencar CRB,Silva OL, Mendonça FL, Andrade FJP. Strategies for control and treatment of carious lesions in deciduous molars: a review of the literature. RGO 2016 Jan-Mar; v.64, n.1.

11. Ludwig KH, Fontana M, Vinson LA, Platt JA, Dean JA. The success of stainless steel crowns placed with the Hall technique: a retrospective study. J Am Dent Assoc. 2014 Dec; 145(12):1248-1253.

12. World Health Organization. Oral health surveys, basic methods. 4th ed. Geneva: 1997.

Recibido: 22/01/2020

Aceptado: 10/12/2020

Correspondencia: Carolina Vansan Martins da Silva, correo: martinsvansan@uol.com.br 